\section{Compression to reduce leg edema in patients with arterial occlusive disease - double focal compression bandaging}

\author{
Carlos Sanchez \\ Primary Care Galego Health Service, \\ Lugo, Spain
}

\section{Introduction}

Usually compression therapy is considered dangerous or contraindicated in patients with leg oedema and arterial occlusive disease.

In my primary care office, I use a Doppler instrument to diagnose arterial occlusive disease by measuring the systolic ankle pressure. Ankle pressure divided by the systolic arm pressure is called the Ankle Brachial Pressure Index (ABPI).

This non-invasive test can indicate $(\mathrm{ABPI}):(1.0-1.4) \rightarrow$ No narrowing or blockage of the leg-arteries; (0.9-1.0) $\rightarrow$ Acceptable state of arteries in legs; (0.8$0.9) \rightarrow$ Some arterial disease; (0.6-0.8) $\rightarrow$ Moderate arterial disease; (less than 0.5 ) $\rightarrow$ Severe arterial disease, critical ischemia.

Guidelines recommend referring the patient to a vascular specialist when this index is less than 0.8 . According to guidelines, compression is strongly contraindicated in severe peripheral arterial disease, when the Ankle Brachial Pressure Index (ABPI), measured by Doppler ultrasound is less than $0.6{ }^{1}$

What happens when the patient has venous and arterial insufficiency? (Figure $1 \mathrm{~A}$ and $\mathrm{B})$.

Can we use compression therapy in a patient with arterial occlusive disease? The following case reports show that compression may be very effective even in such patients, even improving arterial flow, but a careful follow up during the first days/weeks is necessary. ${ }^{2}$

It is possible to reduce edema and heal ulcers in a patient with occlusive arterial disease, using a simple technique of compression therapy, which we call Double focal compression bandaging. The first bandage is used for the focal compression of the wound bed and another bandage covers the first, to achieve a gradual external compression from the toes to the knee., each turn of the band covers the preceding tour by $50-70 \%$. The area of the ulcer receives the pressure of 3 layers (that of the pressure over the wound bed, and the double effect of the external gradual compression). To apply this technique safely in pri- mary care, it is necessary to follow three simple steps: i) To establish a differential diagnosis; ii) To establish a clinical diagnosis; iii) Measurement of the Ankle Brachial Index to exclude severe arterial disease: $\mathrm{A} /$ Hand held Doppler ultrasound device. B/ 5.07 Monofilament and $128 \mathrm{~Hz}$ tuning fork. C/ Scales. D/ The Edinburgh claudication questionnaire. E/ Camera: We take photos of the wound bed at the beginning of treatment, and whenever the bandage is removed, this way, we can observe the clinical course of the ulcer until its healing.

i) We use gauzes to make a padding of a thickness of $1 \mathrm{~cm}$, with a size like that of the ulcer, surpassing its edges. This is what we call focal compression. ii) Adhesive bandage for fixing the padding to the wound bed. iii) Strong inelastic compression bandaging $(10 \times 12 \mathrm{~cm})$ for making the gradual external compression. iv) Physiological saline solution for cleaning the wound bed. v) Adhesive tape for fixing the bandages and scissors to trim the gauze used as focal compression.

Measuring local pressure by Picopress ${ }^{\circledR}$ we found sub-bandage pressure peaks ( $1 \mathrm{~cm}$ padding) over the wound bed, of up to $95 \mathrm{mmHg}$ during walking (inelastic bandaging has better physiological effects on calf muscle pump). ${ }^{3}$

Three clinical cases with severe occlusive arterial disease, who were treated by this technique are presented.

\section{Case Reports}

\section{Case \#1}

Male 49 years old, arterial hypertension, diabetes and dyslipidaemia. Smoker of 40 cigarettes per day. (18-09-2008): Hospital admission because of severe resting pain in both legs, and no peripheral pulses. ANGIOGRAPHY: LericheSyndrome, (bilateral occlusion of distal aorta), no candidate for endovascular therapy. (22-09-2008): Aorto-bifemoral bypass surgery, achieving peripheral pulses in both feet. Eight years later, (20-12-2016): Hospital admission. Resting pain right leg. New angiography: left branch of the aortobifemoral bypass is occluded. Right: Femoro-popliteal occlusion. Thrombectomy right branch, bypass to right deep femoral artery (30-12-2016): The patient was released from hospital. Nine months later (15-09-2017): Readmission to hospital because of critical ischemia of left leg. Occluded abdominal aorta, common and superficial femoral arteries occluded. (17-10-2027): Dismissed with an ulcer over the basis of the left 5 th toe, plantar necrosis
Correspondence: Carlos Sanchez, Primary Care Galego Health Service, Lugo, Spain. E-mail: camesues@gmail.com

Conference presentation: International Compression Club (ICC) Meeting, Rotterdam, 2018

This work is licensed under a Creative Commons Attribution 4.0 License (by-nc 4.0).

(C) Copyright C. Sanchez, 2018

Licensee PAGEPress, Italy

Veins and Lymphatics 2018; 7:7980

doi:10.4081/vl.2018.7980

over 2nd toe, swelling of foot and leg. Left 5 th toe was amputated.

This patient was treated with compression therapy, two and a half month later the ulcer and the necrosis were healed, oedema was reduced.

ABPI before compression $=0.70 /$ ABPI after compression $=1.06$

\section{Case \#2}

Male 56 years old, arterial hypertension, dyslipidaemia, Ex-smoker goes to the hospital because of severe pain at rest, in the forefoot and first toe of the left foot. Angiograhy: Popliteal artery occlusive disease. (25-03-2011): Bilateral femoropoplitealobliteration. Cardiac catheterization bypass surgery. (6-04-2011): Trans metatarsal amputation of the left foot. (2603-2014): Left $\mathrm{ABI}=0.60$; Right $\mathrm{ABI}=$ 0.65. (27-10-2014): Left $\mathrm{ABI}=0.58$; Right $\mathrm{ABI}=0.68 .(26-07-2016)$ : Cardiac catheterization shows coronary disease. Percutaneous revascularization. (11-082016): The patient was released from hospital.

Cardiologists ask for interconsultation with angiologist to evaluate its peripheral artery disease. Angiologist detect very weak pulses in left leg. (16-08-2016): We started treating the patient with inelastic bandages and recommended walking exercises, making a daily follow- up, in the first weeks. We teach the patient to bandage the leg, achieving to increase the ABPI in both legs: 4 months later $($ Left $=0.72$ : Right $=0.89) .10$ months later: $($ Left $=0.94:$ Right $=0.98) .16$ months later $($ Left $=0.98$ : Right $=1.02)$.

The ABPI was increased and the pain and oedema decreased.

\section{Case \#3}

Female 72-year-old, arterial hypertension. type 2 diabetes mellitus, arterial ulcer and oedema of the left leg. Angiography showed multisegment arterial occlusive disease, not amenable to surgical revasculari- 

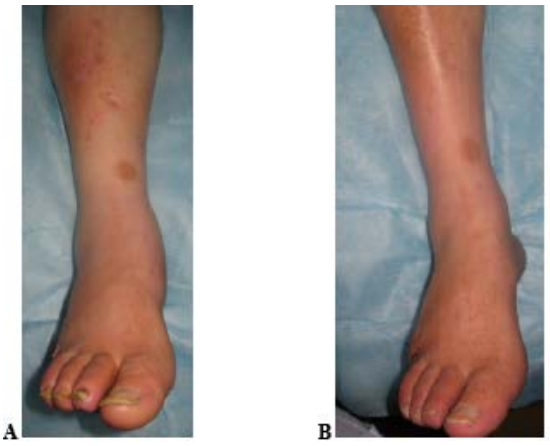

Figure 1. A) Before compression; B) Three months later compression bandaging.

sation. After 4 months of wearing inelastic bandages, with increased pressure over the ulcer area and hypertension and diabetes under control, the patient was pain-free and the ulcer had healed. The ankle-brachial pressure index (ABI) increased from 0.54 at the start of treatment to 0.70 . We followed with compression therapy (double focal compression bandaging) and achieved to increase the ABPI. At the moment (Left $\mathrm{ABI}=1.14 /$ Right $\mathrm{ABI}=1.19$ ).

\section{Conclusions}

Compression is strongly contraindicated in the event of severe peripheral arterial disease, when the Ankle Brachial Pressure Index (ABPI), measured by Doppler ultrasound is less than 0.6, However, in expert hands and according to signs and symptoms, making a daily follow up, it is possible and convenient applying compression therapy, for reducing leg swelling and improving arterial flow in cases, in whom surgical revascularisation cannot be for improving arterial flow in cases, in whom surgical revascularisation cannot be performed. This is demonstrated in three cases by an increase of ABPI suggesting an improvement of the collateral circulation due to the massaging effect of inelastic compression together with walking exercises. ${ }^{4,5}$

\section{References}

1. O'Donnell TF Jr, Passman MA, Marston WA, et al. Management of venous leg ulcers: clinical practice guidelines of the Society for Vascular Surgery ${ }^{\circledR}$ and the American Venous Forum. J Vasc Surg 2014;60:3S-59S.

2. Sanchez C, Partsch H. Healing of an arterial ulcer by compression bandaging. A case report. J Wound Care WUWHS Suppl 2017;26:2.

3. Mosti G, Iabichella ML, Partsch H. Compression therapy in mixed ulcers increases venous output and arterial perfusion. J Vasc Surg 2012;55:122-8.

4. Alvarez OM, Wendelken ME, Markowitz L, Comfort C. Effect of high-pressure, intermittent pneumatic compression for the treatment of peripheral arterial disease and critical limb ischemia in patients without a surgical option. Wounds 2015;27:293-301.

5. Labropoulos N, Leon LR Jr, Bhatti A, et al. Hemodynamic effects of intermittent pneumatic compression in patients with critical limb ischemia. J Vasc Surg 2005;42:710-6. 